\title{
Experimental verification of the physical nature of velocity-stress relationship for isotropic
} porous rocks

\author{
Marina Pervukhina ${ }^{l}$, Boris Gurevich ${ }^{* 1,2}$, David N. Dewhurst $^{l}$ and Anthony F. Siggins ${ }^{3}$ \\ ${ }^{1}$ CSIRO Petroleum, Kensington, Australia.E-mail: marina.pervukhina@csiro.au \\ ${ }^{2}$ Curtin University of Technology, Department of Exploration Geophysics, Kensington, Australia \\ ${ }^{3}$ CSIRO Petroleum, Clayton, Australia
}

\section{Summary}

Exponential increase of seismic velocities with effective stress has usually been explained by presence of pores with a broad distribution of aspect ratios. More recently, a stress-related closure of soft pores with a narrow distribution of compliances has been suggested as an alternative explanation of such exponential stress dependency. This theoretical interpretation has been verified here using laboratory measurements on dry sandstones. On the basis of these experimental data, linear dependency of elastic compressibility on soft porosity and exponential decay of soft porosity and elastic compressibility with effective stress up to $60 \mathrm{MPa}$ is confirmed. Soft porosity, estimated from the fitting coefficients of elastic compressibilities, is on the same order of magnitude but slightly lower than obtained from strain measurements. The results confirm applicability of previously proposed stress sensitivity model and provide justification for using this approach to model stress dependency of elastic properties.

\section{Introduction}

Knowledge of stress dependency of elastic properties of rocks is important for a variety of geophysical applications ranging from pore pressure prediction in sedimentary rocks and seismic monitoring of hydrocarbon production to constraining material properties in the mantle. It has been shown by many authors that stress dependency of compressional and shear velocity $V$ in many dry porous rocks can be well approximated by a combination of linear and exponential terms (e.g., Zimmerman et al., 1991; Eberhart-Phillips et al., 1989):

$$
V(P)=A+K P-B \exp (-P D),
$$

where $P$ is confining pressure, $A, K, B$, and $D$ are fitting parameters that provide the best agreement with the measured data.

Until recently, the exponential term in equation (1) was usually explained by presence of pores and cracks with broad (usually exponential) distribution of crack stiffnesses or aspect ratios (e.g., Zimmerman, 1991; Tod, 2002). Recently, Shapiro (2003) obtained a somewhat surprising result that such exponential form may be explained by much more simple dual distribution of porosity. More precisely, Shapiro (2003) showed that if total porosity $\phi$ of an isotropic rock can be divided into stiff porosity part $\phi_{s}$ and much more compliant part $\phi_{c}$, i.e.

$$
\phi=\phi_{s}+\phi_{c},
$$

then dry rock compressibility $C_{d r}$ can be written as a simple linear function of stiff and compliant porosities

$$
C_{d r}(P)=C_{d r s}\left(1+\theta_{s} \Delta \phi_{s}+\theta_{c} \phi_{c}\right)
$$

where $C_{d r s}$ is the compressibility of the porous rock with all compliant porosity closed (hereafter, stiff limit), $\theta_{s}$ and $\theta_{c} \gg \theta_{s}$ are coefficients related to compliance of stiff and compliant pores, respectively, $\Delta \phi_{s}=\phi_{s}-\phi_{s 0}$ is the deviation of the stiff porosity from its zero-pressure value $\phi_{s 0}$. Note a change in notation: $\Delta \phi_{s}$ in the present work corresponds to $\phi_{s}$ in Shapiro (2003). In many cases, $\theta_{s} \Delta \phi_{s}<<\theta_{c} \phi_{c}$, and equation (3) reduces to

$$
C_{d r}(P)=C_{d r s}\left(1+\theta_{c} \phi_{c}\right),
$$

Variations of stiff and soft porosity with pressure have the form

and

$$
\Delta \phi_{s}=P\left[C_{g r}-\left(1-\phi_{s 0}\right) C_{d r s}\right]
$$

$$
\phi_{c}=\phi_{c 0} \exp \left(-\theta_{c} C_{d r s} P\right),
$$

where $C_{g r}$ is the compressibility of the solid grain material and $\phi_{c 0}$ is the compliant porosity at zero pressure. Note that equation (5) is slightly more general than the form used by Shapiro (2003), as it does not require initial stiff porosity $\phi_{s 0}$ to be small. Substituting expression (6) for compliant porosity into equation (4) gives the variation of compressibility with pressure $\Delta C_{d r}(P)=C_{d r}(P)-C_{d r s}$ (deviation of dry compressibility $C_{d r}(P)$ from its stiff limit $C_{d r s}$ ) in the form:

$$
\Delta C_{d r}(P)=C_{d r s} \theta_{c} \varphi_{c 0} \exp \left(-\theta_{c} C_{d r s} P\right) .
$$

If this variation is small ( $\Delta C_{d r}(P)<<C_{d r s}$ ), then equation (3) together with the corresponding equation for the shear compliance yields equations similar in form to (1) for the bulk and shear moduli and for compressional and shear velocities of the dry rock (Shapiro, 2003). The moduli and velocities of saturated rocks (at low frequencies) can be obtained using Gassmann equations; in this case pressure $P$ has the meaning of effective pressure. 


\section{Experimental verification of the physical nature of velocity-stress relationship for isotropic porous rocks}

While the stress sensitivity approach of Shapiro (2003) provides an appealingly simple interpretation of equation (1), it is not yet widely accepted, partly due to the lack of the experimental verifications of this interpretation. In this paper we overcome this difficulty by using simultaneous measurements of ultrasonic velocity and porosity in a high pressure cell. Porosity variations are estimated from measurements of axial strain. The results of our analysis for the sandstone samples used in the experiments are consistent with the physical interpretation of Shapiro (2003).

\section{Workflow}

Our workflow consists of three main parts: laboratory measurements, computation of the key parameters, and analysis.

\section{Experiment}

The experimental program involves the following steps

1. Measure compressional $V_{p}$ and shear $V_{s}$ velocities for a dry sample as a function of confining hydrostatic pressure $P$.

2. Simultaneously measure axial strain $\varepsilon_{a x}$ as a function of pressure.

3. Measure initial porosity at zero pressure $\phi_{0}$ and/or initial density $\rho_{0}$.

\section{Calculations of key parameters}

Once these data are measured, compressibilities and stiff and soft porosities are obtained as follows.

4. For each pressure, the dry bulk modulus $K_{d r}$ is obtained using the standard equation $K_{d r}=\left[V_{p}^{2}-(4 / 3) V_{p}^{2}\right] \rho_{0}$ and compressibility as $C_{d r}=K_{d r}^{-1}$. Note that since variation of velocities with pressure is always much larger than that of density, calculation of the bulk modulus using initial density $\rho_{0}$ at $P=0$ is sufficiently accurate.

5. Then, we compute variation of porosity with pressure. Since experiments are performed with hydrostatic pressure, we assume that $\varepsilon_{a x}$ represents the strain in all three directions. Then, the volumetric strain (relative variation of the sample volume $v$ with pressure) is $\mathcal{E}=\left(v-v_{0}\right) / v_{0}=3 \varepsilon_{a x}$, where $v_{0}$ is sample volume at zero pressure. At the same time, the relative change of total volume $v_{g r}$ of solid grains with pressure is $\varepsilon_{g r}=\left(v_{g r}-v_{g r 0}\right) / v_{g r 0}=-C_{g r} P /(1-\phi)$, where $v_{g r 0}$ is total volume of solid grains at zero pressure and $C_{g r}$ is the compressibility of the solid grain material, which is assumed known from mineralogical analysis.
Since pore volume at any pressure is the total volume minus grain volume, $v_{\phi}=v-v_{g r}$, using Zimmerman et al. (1986), we get:

$$
\phi=\frac{v_{\phi}}{v}=1-\frac{v_{g r}}{v}=1-\frac{\left[1-C_{g r} P /(1-\phi)\right] v_{g r 0}}{(1+\varepsilon) v_{0}} .
$$

For small deformations, this gives

$$
\phi=1-(1-\varepsilon)\left(1-\phi_{0}\right)-C_{g r} P=\phi_{0}+\left(1-\phi_{0}\right) \varepsilon+C_{g r} P .
$$

Note that for $P>0$, total volumetric strain, $\varepsilon$, is negative, and is larger in absolute value than the grain deformation $C_{g r} P$; therefore, using (9), porosity will decrease with increasing pressure, as it should.

6. Now we have to define the parameters which we called stiff and soft porosities. Since stiffness of pores is a relative measure, there is some freedom inherent in this distinction. One way to define stiff porosity is to assume that within the pressure range of measurements, say from 0 to $100 \mathrm{MPa}$, the stiff porosity changes linearly with pressure. Indeed, if measured compressibilities and velocities can be described by a simple exponential relationship of the type given by equation (4), then at large pressures the compressibility $C_{d r}$ approaches a constant value $C_{d r s}$. Constant (pressure independent) compressibility corresponds to a linear variation of the strain with pressure. Indeed, in a pressure interval where dry compressibility is approximately constant, $C_{d r} \approx C_{d r s}$, volumetric strain $\varepsilon=-C_{d r} P$ and equation (8) gives a linear relationship between porosity and pressure and indicate equation (5). This linear porosity trend is expected in the upper part of our pressure range, where all soft pores can be assumed closed. We can thus call this part of the porosity the stiff porosity. Assuming, following Shapiro (2003), that stiff and soft porosity variations are independent of one another, we can extrapolate this stiff porosity trend to lower pressures. In practice, we will estimate stiff porosity by fitting a linear trend to the porositypressure dependency in the uppermost part of the measurement pressure range (Walsh, 1965; Mavko and Jizba, 1991).

7. Once stiff porosity is defined, the compliant (soft) porosity is defined by equation (2) as the difference between total and stiff porosity. We will also obtain soft porosity from a non-linear fitting of experimentally measured $\Delta C_{d r}(P)$ as a ratio of the coefficient before the exponential to the exponent as predicted by equation (7). Hereafter, we refer to the former and later soft porosity as measured and predicted, respectively. 


\section{Experimental verification of the physical nature of velocity-stress relationship for isotropic porous rocks}

In this study we use non-linear Levenberg-Marquardt algorithm for approximation of all exponential dependencies.

Testing the theoretical predictions

With equations (2) and (5) used effectively as definitions, we will test experimentally the following elements of Shapiro's (2003) stress sensitivity theory:

8. Test the adequacy of the linear relationship (4) between the dry compliance and soft porosity.

9. Test the adequacy of the exponential approximation, equation (6), for the soft porosity.

10. Compare the exponent $\lambda_{\phi}$ in the soft porosity fit, equation (6), with the exponent $\lambda_{C}$ in the $\Delta C_{d r}$ fit , equation (7).

11. Compare the measured against predicted soft porosity.

\section{Data}

We used reservoir sandstone core samples from the Northwest Shelf of Australia between depths of 1700-2000 $\mathrm{m}$ to validate the stress dependency model. Cores for testing were cut with diameters of $38 \mathrm{~mm}$ and length of 76 $\mathrm{mm}$. Lithologies were homogeneous over the length of the cores. The porosity ranged from $7-24 \%$ with an average clay content of $4 \%$. The sandstones were tested dry and oil saturated under ambient conditions in a triaxial cell to a maximum differential pressure of $60 \mathrm{MPa}$. More details of the sandstones and experimental method can be found in Siggins and Dewhurst (2003).

We calculate elastic compressibilities using longitudinal and shear velocities in the dry samples. As an example, Figure 1a shows the stress dependency of dry elastic compressibility for one of the samples (sample12/04 from Siggins and Dewhurst (2003)) for loading (red circles) and unloading (blue squares) processes. The experimentally measured compressibilities exponentially decrease with effective pressure; exponential fits for loading and unloading are shown by solid red and dashed blue lines, respectively.

Porosity variations are obtained from the variations of the length of the cylindrical samples. Total, stiff and compliant porosity are shown in Figure $1 \mathrm{~b}$ for the same sample for loading (in red) and unloading (in blue). As outlined above, stiff porosity (dashed lines) is obtained by linearly extrapolation of the high-stress trend determined from two points at 50 and $60 \mathrm{MPa}$. Compliant porosity (diamonds) is estimated by subtracting stiff porosity from the total porosity and is reasonably approximated by an exponential fit (dotted lines). The linear plus exponential fits for total porosity are shown as solid lines.

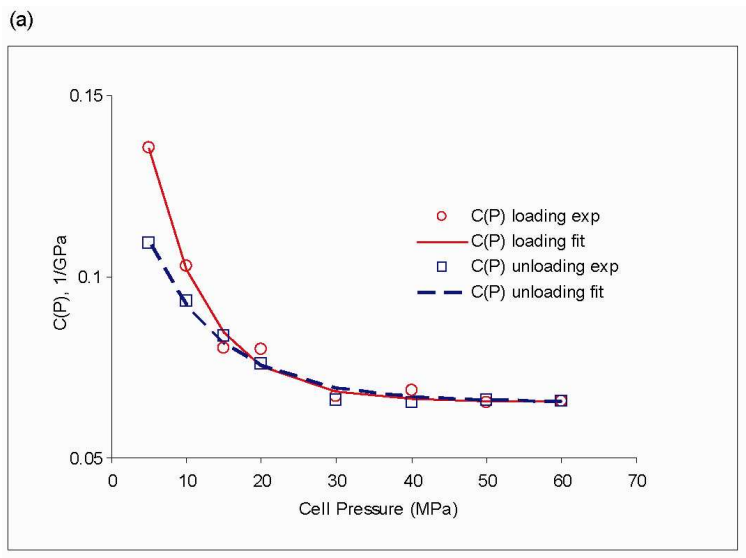

(b)

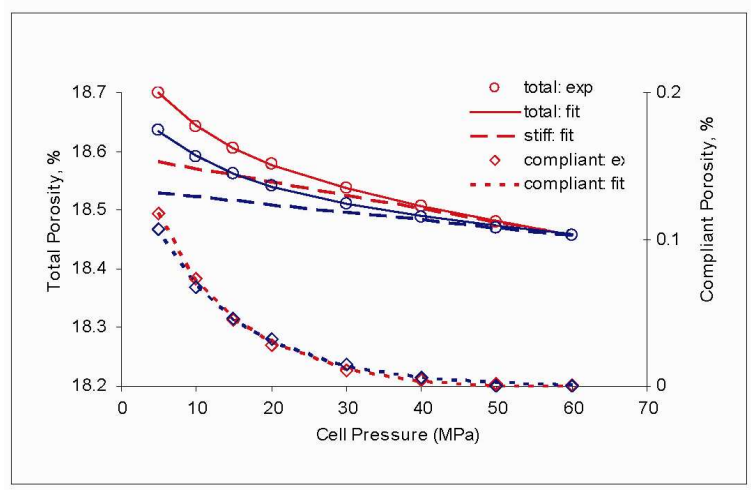

Figure 1: Experimental measurements and fitting of stress dependency of (a) elastic compressibility and (b) stiff, soft and total porosity in a dry sandstone. Experimental points and fitting curves for loading and unloading are shown in red and blue, respectively.

\section{Results}

First, in Figures 2 and 3 we illustrate tests of the stress sensitivity theory for one sample (sample 12/04), and then show test results on all samples (Figure 4). The test of linearity of the relationship between compressibility and soft porosity (equation (4)) is demonstrated in Figure 2, which shows pressure variation of compressibility $\Delta C_{d r}(P)$ as a function of compliant porosity and its linear fits for loading and unloading limbs. The square of the Pearson product moment correlation coefficients are 0.98 and 0.97 , respectively, indicating a good correlation between $\Delta C_{d r}(P)$ and $\varphi_{c}(P)$.

Measured stress dependencies of variations of elastic compressibility $\Delta C_{d r}$ and compliant porosity $\varphi_{c}$ with 


\section{Experimental verification of the physical nature of velocity-stress relationship for isotropic porous rocks}

stress for the same sample 12/04 are presented in logarithmic scale in Figure 3. Straight solid and dotted lines show linear fits of the compressibility variations and compliant porosity, respectively. The linear fits are nearly parallel for compressibility deviation $\Delta C_{d r}(P)$ and soft porosity $\varphi_{c}(P)$. This confirms that compressibility variation and soft porosity are well approximated by the same exponentials.

Comparison of compressibility variation exponent $\lambda_{C}$ vs. soft porosity exponent $\lambda_{\phi}$ for all the samples is shown in Figure 4a. Exponents can be fitted with trends of $\lambda_{C}=1.07 \lambda_{\phi}$ and $\lambda_{C}=0.96 \lambda_{\phi}$ for loading and unloading, respectively.

Predicted soft porosities shown in Figure $4 b$ in comparison with measured ones are of the same order of magnitude but somewhat lower. The predicted vs. measured soft porosity trends are $\theta_{p r}=0.74 \theta_{m}$ and $\theta_{p r}=0.84 \theta_{m}$ for loading and unloading, respectively, indicating a better prediction of the soft porosity from the measured compressibility data at unloading.

\section{Conclusions}

Theoretical interpretation of exponential stress dependency of elastic properties of rocks based on a dual porosity concept has been verified using laboratory measurements on dry sandstones. On the basis of the experimental data, the following postulates of the theoretical model are confirmed: (1) linear dependency of elastic compressibility on soft porosity and (2) exponential decay of soft porosity and elastic compressibility with effective stress up to 60 $\mathrm{MPa}$. Magnitude of the variation of stiff porosity with stress is shown to be comparable with compliant porosity. However, this variation has a negligible effect on rock compressibility up to $60 \mathrm{MPa}$. Soft porosity estimated from the fitting coefficients of elastic compressibilities is on the same order of magnitude but slightly lower than obtained from strain measurements. These results confirm applicability of Shapiro's (2003) stress sensitivity model and hence provide justification for using this approach to model the effect of stress on properties of not only for isotropic, but also anisotropic rocks.

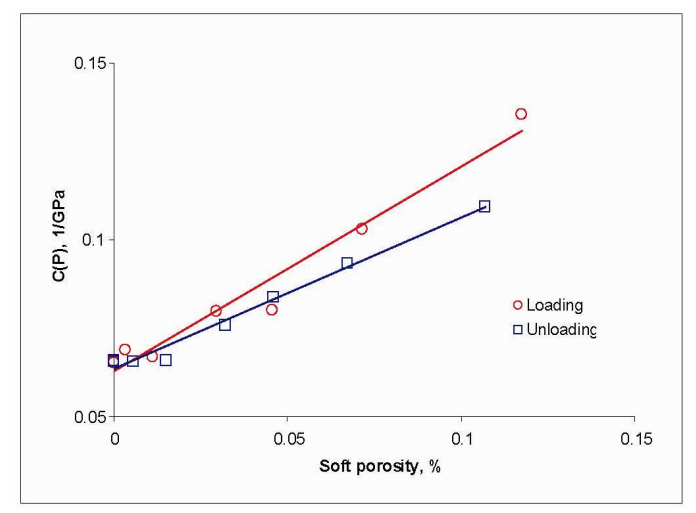

Figure 2. Elastic compressibilities vs. soft porosity and linear trends for loading and uploading processes. Colours and markers have the same meaning as in Figure 1.

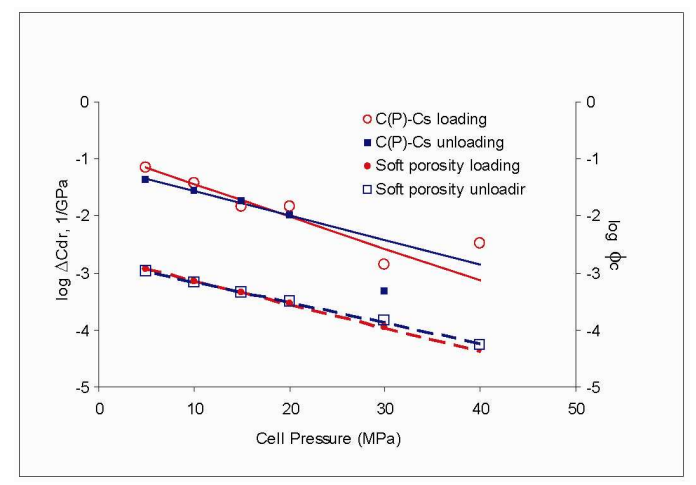

Figure 3. Measured stress dependencies of decimal logarithms of variations of elastic compressibility $\Delta C_{d r}$ and compliant porosity $\phi_{c}(P)$ with stress. Straight solid and dotted lines show exponential fitting of the compressibility variations and compliant porosity, respectively.
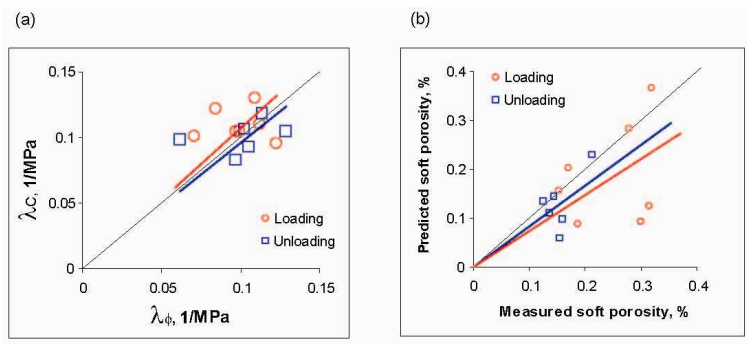

Figure 4. Comparison of (a) compressibility vs. soft porosity exponents, (b) predicted and measured soft porosities and (c) dynamic and static stress sensitivity coefficients obtained for all the sandstone samples. 


\section{EDITED REFERENCES}

Note: This reference list is a copy-edited version of the reference list submitted by the author. Reference lists for the 2009 SEG Technical Program Expanded Abstracts have been copy edited so that references provided with the online metadata for each paper will achieve a high degree of linking to cited sources that appear on the Web.

\section{REFERENCES}

Eberhart-Phillips, D., D. H. Han, and M. D. Zoback, 1989, Empirical relationships among seismic velocity, effective pressure, porosity and clay content in sandstone: Geophysics, 54, 82-89.

Mavko, G., and D. Jizba, 1991, Estimating grain-scale fluid effects on velocity dispersion in rocks: Geophysics, 56, 1940-1949.

Shapiro, S. A., 2003, Elastic piezosensitivity of porous and fractured rocks: Geophysics, 68, 482-486.

Siggins, A. F., and D. N. Dewhurst, 2003, Saturation, pore pressure and effective stress from sandstone acoustic properties: Geophysical Research Letters, 30, No. 2, doi: 10.1029/2002GL016143.

Tod, S. R., 2002, The effects of stress and fluid pressure on the anisotropy of interconnected cracks: Geophysical Journal International, 149, 149-156.

Walsh, J., 1965, The effect of cracks on the compressibility of rock: Journal of Geophysical Research, 70, 381-389.

Zimmerman, R. W., 1991, Compressibility of sandstones: Elsevier.

Zimmerman, R. W., W. H. Somerton, and M. S. King, 1986, Compressibility of porous rocks: Journal of Geophysical Research, 91, 12765-12777. 